\title{
NÍVEIS PLASMÁTICOS DE TAURINA E DE SEUS PRECURSORES EM PACIENTES COM CÂNCER DE ESÔFAGO
}

\author{
Vânia Cristina LAMÔNICA-GARCIA', Flávia Andréa MARIN¹, Mauro Masson LERCO², \\ Fernando MORETO ${ }^{3}$, Maria Aparecida Coelho Arruda HENRY2 e Roberto Carlos BURINI ${ }^{4}$
}

\begin{abstract}
RESUMO - Racional - O câncer de esôfago tem impacto relevante no metabolismo protéico do hospedeiro, mas pouco se conhece sobre as implicações no metabolismo protéico sulfurado. Deste, destaca-se a taurina, composto participante de várias funções fisiológicas importantes como a manutenção do sistema de defesa celular e possível sobrevida do paciente. Objetivo - Estudar as variações plasmáticas da taurina e de seus precursores em pacientes com câncer de esôfago. Método - Em estudo transversal foram triados 16 pacientes (43-73 anos) com câncer de esôfago e 20 voluntários (27-65 anos) controles sadios que preencheram os critérios clínicos e éticos da pesquisa. Para caracterização do estado geral de saúde efetuou-se avaliação antropométrica, hematimétrica (Hb, Ht, glóbulos brancos, linfócitos) e bioquímica (albumina, glicose, lipídios, aminotransferases). Adicionalmente, foram realizadas, no plasma, análises cromatográficas de taurina e seus precursores cisteína e homocisteína. Foi registrado o tempo de sobrevivência dos pacientes, a partir do diagnóstico histopatológico. Resultados - Os pacientes com câncer de esôfago foram predominantemente do sexo masculino, raça branca, classe socioeconômica baixa, tipo carcinoma espinocelular de localização no terço superior, em estádio IV, sobrevida de 7,8 $\pm 5,5$ anos, referindo perda de peso em $16,4 \%$ e apresentando hipoalbuminemia em $50 \%$, com massa muscular e adiposa semelhante ao controle. Os pacientes apresentaram valores estatisticamente menores do que os controles para $\mathrm{Hb}, \mathrm{Ht}$, colesterol total, HDL-colesterol e cisteína e maiores de AST, ALT, taurina e homocisteína. Dentre os pacientes houve correlação positiva da taurina tanto com a contagem total de linfócitos, como com a sobrevida dos pacientes. Conclusão - Os níveis reduzidos de cisteína e elevados de homocisteína, taurina e as associações positivas da taurina com os indicadores da imunocompetência celular e da mortalidade sugerem participação efetiva da taurina na sobrevida dos pacientes e, portanto, os cuidados nutricionais específicos com a sua via geradora (cisteína, metionina e vitaminas do complexo B)
\end{abstract}

DESCRITORES - Neoplasias esofágicas. Taurina.

\section{INTRODUÇÃO}

O câncer de esôfago (CE) é uma doença cuja resultante desnutrição protéico-energética constitui um dos principais fatores de risco, devido à diminuição da ingestão de alimentos e às alterações metabólicas promovidas pelo crescimento tumoral. Tal enfermidade é mais freqüente em pessoas do sexo masculino, com idade acima de 50 anos, e está intimamente relacionada ao consumo excessivo de álcool e tabaco ${ }^{(4)}$.

Tendo em vista que o CE se constitui em doença de prognóstico desfavorável, com grandes implicações metabólico-nutricionais, é de grande importância entender sua patogênese e desenvolver estratégias para a prevenção e melhoria da sobrevivência de pacientes acometidos desse mal.

Há evidências de que a regulação de citocinas próinflamatórias e a estimulação de citocinas antiinflamatórias poderiam favorecer uma resolução mais eficiente da resposta inflamatória em pacientes com $\mathrm{CE}$, prevenindo o aparecimento de complicações e melhorando sua sobrevivência. Esses benefícios podem estar relacionados com as ações da taurina ${ }^{(1,8,19)}$.

Existem poucos trabalhos estudando os níveis de taurina plasmática em humanos com câncer, principalmente com neoplasias malignas de esôfago ${ }^{(8,31)}$.

A taurina é um aminoácido livre, abundante no meio intracelular, proveniente do metabolismo da metionina, sendo indispensável para felinos e condicionalmente indispensável em humanos. Isso porque seus níveis plasmáticos diminuem em determinadas situações de estresse metabólico como sepse, traumas e cirurgias. Curiosamente, esse aminoácido não é incorporado a outras proteínas por não ter o RNA mensageiro específico $^{(24)}$.

A síntese desse aminoácido ocorre por derivação da metionina e cisteína, portanto sem recomendações estabelecidas (RDA -Recommended Daily Allowance) para humanos ${ }^{(11)}$.

'Programa de Pós-graduação em Bases Gerais da Cirurgia; ${ }^{2}$ Departamento de Cirurgia e Ortopedia; ${ }^{3}$ Programa de Pós-graduação em Patologia; ${ }^{4}$ Departamento de Saúde Publica e Centro de Metabolismo em Exercício e Nutrição, Faculdade de Medicina da Universidade Estadual Paulista, Botucatu, SP.

Correspondência: Nutr. Vânia C. L. Garcia - Rua João Croce, 1-65 - Jardim Shangrilá - 17054-638 - Bauru, SP. E-mail: vanialamonica@uol.com.br 
Suas funções principais são osmorregulação, modulação do cálcio iônico, estabilização da membrana plasmática, detoxificação dos ácidos biliares, desenvolvimento do sistema nervoso central e da retina, neurotransmissor inibitório, imunomodulação e atividade antioxidante, diminuição da formação do ácido hipocloroso $\left(\mathrm{HOCl}^{-}\right)$na eclosão fagocitária e, também, possível modulação das citocinas pró-inflamatórias ${ }^{(22,23,24)}$.

A mieloperoxidase (MPO) é uma enzima liberada dos neutrófilos durante a eclosão respiratória no processo inflamatório ${ }^{(25)}$; sua combinação com peróxido de hidrogênio $\left(\mathrm{H}_{2} \mathrm{O}_{2}\right)$, proveniente da ação do superóxido, e o cloro $\left(\mathrm{Cl}^{-}\right)$formam o $\mathrm{HOCl}^{-}$, agente bactericida altamente deletério. No entanto, sua toxicidade não é restrita às membranas bacterianas, danificando também as células do hospedeiro(26). Os leucócitos, quando ativados, utilizam o sistema MPO- $\mathrm{H}_{2} \mathrm{O}_{2}-\mathrm{Cl}$, juntamente com a taurina, para formar taurocloramina, composto de menor citotoxicidade do que o $\mathrm{HOCl}^{-}$. Assim, a taurina tem papel importante na preservação da integridade dos leucócitos pela competição pelo $\mathrm{HOCl}^{-(22,25)}$.

Trabalhos in vitro ${ }^{(5,18,21,25)}$ mostram que as concentrações de taurina, nos neutrófilos, linfócitos e leucócitos, são extremamente altas. Há evidências de que essas concentrações ocorram em virtude de possível modulação da função imune, pela preservação da habilidade fagocítica dos neutrófilos, por meio da interação com a enzima $\mathrm{MPO}^{(22,23)}$. Não foram encontrados trabalhos similares in vivo, em humanos.

Considerando o exposto, este estudo se propôs a estudar o metabolismo da taurina em pacientes com CE.

\section{MÉTODO}

Foram triados 36 indivíduos, sendo 16 pacientes com CE (grupo 1 - G1) e 20 indivíduos controle (grupo 2 - G2), no período de janeiro a dezembro de 2004 . O G1 foi composto por duas mulheres e 14 homens com média de idade de 59,7 $\pm 9,9$ (43-73) anos, com diagnóstico de CE admitidos na enfermaria da gastrocirurgia do Hospital das Clínicas da Faculdade de Medicina de Botucatu - Universidade Estadual Paulista - UNESP, Botucatu, SP. Para o grupo controle (G2) foram selecionados 10 mulheres e 10 homens, voluntários, não-fumantes, nãoalcoólatras, não-consumidores de suplementos nutricionais, sem história prévia de neoplasias, com idade média de 37,2 $\pm 9,8$ (27-65) anos. Todos os participantes foram informados sobre os procedimentos da pesquisa e assinaram o Termo de Consentimento Livre e Esclarecido aprovado pelo Comitê de Ética em Pesquisa local. Após o diagnóstico de CE e estádio de acordo com a classificação dos tumores malignos TNM (International Union Against Cancer - UICC,1987), foi realizada avaliação do estado nutricional (bioquímica e antropométrica) e colhido sangue para análise dos aminoácidos sulfurados (taurina, cisteína e homocisteína) pelo método de Cromatografia Líquida de Alta Eficiência (HPLC) ${ }^{(10,27)}$, antes de qualquer intervenção clínica ou nutricional. O método consistiu na avaliação clínica por meio de endoscopia e biopsia da lesão esofágica. Após a confirmação de neoplasia maligna, procedeu-se à broncoscopia e tomografia tóraco- abdominal para se efetuar o estádio. A caracterização nutricional envolveu aspectos antropométricos e bioquímicos. A avaliação antropométrica foi composta pela percentagem de perda de peso (\%PP), índice de massa corporal (IMC), percentagem de gordura corporal (\%GC) e circunferência muscular do braço (CMB). A avaliação bioquímica constou de contagem total de linfócitos (CTL), albumina plasmática, glicemia, hematócrito, hemoglobina, colesterol total e frações, triglicerídios e aminotransferases. Foram considerados valores normais para taurina plasmática os compreendidos entre $25-100 \mu \mathrm{mol} / \mathrm{L}^{(11)}$; para homocisteína entre $5-15 \mu \mathrm{mol} / \mathrm{L}^{(28)}$ e, para cisteína, consideraram-se aceitáveis os valores entre $110,4-229,6 \mu \mathrm{mol} / \mathrm{L}^{(16)}$.

Os dados foram analisados por meio do teste $t$ de Student e correlação de Pearson.

\section{RESULTADOS}

No G1 houve predominância da raça branca, sexo masculino, maior freqüência de etilistas e tabagistas. A localização mais freqüente do tumor foi no terço superior e o tipo histológico predominante foi carcinoma espinocelular. A maioria dos pacientes, no momento do diagnóstico, apresentava estádio IV. A sobrevida foi em média de 7,8 $\pm 5,5$ meses, sendo que $56,2 \%$ sobreviveram mais de 6 meses após o diagnóstico de câncer. As intervenções terapêuticas mais freqüentes realizadas após o diagnóstico e estádio foram as ostomias - jejunostomia $(\mathrm{n}=6)$ e gastrostomia $(\mathrm{n}=3)$ - nove pacientes $(50 \%)$ e cinco $(27,7 \%)$ foram submetidos a radioterapia e quatro $(22,2 \%)$ sofreram esofagectomia/gastroplastia.

A avaliação antropométrica evidenciou que os pacientes estavam em processos de desnutrição, visto que $87,5 \%$ deles apresentaram \%PP acima de $10 \%$, bem como, $75 \%$ mostraram CMB abaixo dos padrões de normalidade e em 52,3\% a \%GC abaixo dos valores esperados. No entanto, o IMC sugeriu eutrofia em $62,5 \%$ dos pacientes. No grupo G2 houve maior freqüência de indivíduos com excesso de peso, com aumento de adiposidade corpórea e redução da massa muscular nas mulheres.

A distribuição dos valores das dosagens plasmáticas dos aminoácidos sulfurados dos grupos de estudo e controle podem ser observados na Tabela 1.

Houve diferença estatística $(P<0,05)$ entre as variáveis: CTL, hemoglobina, colesterol total, HDL-colesterol, albumina, aminotransferases (AST e ALT) e os aminoácidos sulfurados taurina, cisteína e homocisteína (Tabela 2).

Adicionalmente, observou-se que os pacientes que sobreviveram mais que 6 meses, apresentaram concentrações de taurina maiores que nos pacientes que sobreviveram menos que 6 meses $(167,5 \pm 75,2 \mu \mathrm{mol} / \mathrm{L}$ e $67,1 \pm 29,5 \mu \mathrm{mol} / \mathrm{L}$, respectivamente).

A taurina e os demais aminoácidos sulfurados não se correlacionaram com nenhuma das variáveis (bioquímicas e antropométricas) no grupo controle. No grupo de pacientes com câncer de esôfago, no entanto, houve correlação significativa positiva entre a taurina e a CTL $(\mathrm{r}=0,50$ e $P=0,03)$ (Figura 1), assim como entre a taurina e a sobrevivência $(\mathrm{r}=0,71 \mathrm{e}$ $P=0,0001)$ (Figura 2). 
TABELA 1. Valores das dosagens plasmáticas dos aminoácidos sulfurados dos grupos de estudo e controle

\begin{tabular}{|c|c|c|c|c|c|c|}
\hline & \multicolumn{2}{|c|}{ Variáveis } & \multicolumn{4}{|c|}{ Resultados } \\
\hline & G1 & $\mathrm{G} 2$ & \multicolumn{2}{|c|}{ G1 } & \multicolumn{2}{|c|}{ G2 } \\
\hline & MD & /DP & $\mathrm{n}$ & $\%$ & $\mathrm{n}$ & $\%$ \\
\hline $\begin{array}{l}\text { Taurina }(25- \\
100 \mu \mathrm{mol} / \mathrm{L})\end{array}$ & $123,6 \pm 7,5$ & $51,1 \pm 10,1$ & & & & \\
\hline $25-100$ & & & 7,0 & 13,8 & 20,0 & 100,0 \\
\hline$>100$ & & & 9,0 & 56,2 & & \\
\hline Total & & & 16,0 & 100,0 & 20,0 & 100,0 \\
\hline $\begin{array}{l}\text { Cisteína } \\
(110,4-229,6 \\
\mu \mathrm{mol} / \mathrm{L})\end{array}$ & $210,5 \pm 57,5$ & $278,0 \pm 35,1$ & & & & \\
\hline$<110,4$ & & & 1,0 & 6,3 & & \\
\hline $110,4-229,6$ & & & 12,0 & 75,0 & & \\
\hline$>229,6$ & & & 3,0 & 18,8 & 20,0 & 100,0 \\
\hline Total & & & 16,0 & 100,1 & 20,0 & 100,0 \\
\hline $\begin{array}{l}\text { Homocisteína } \\
(5-15 \mu \mathrm{mol} / \mathrm{L})\end{array}$ & $18,7 \pm 7,3$ & $12,1 \pm 3,0$ & & & & \\
\hline $5-15$ & & & 6,0 & 37,5 & 14,0 & 70,0 \\
\hline$>15$ & & & 10,0 & 62,5 & 6,0 & 30,0 \\
\hline Total & & & 16,0 & 100,0 & 20,0 & 100,0 \\
\hline
\end{tabular}

\section{DISCUSSÃO}

No geral, a amostra estudada mostrou-se semelhantes às de outros estudos, com a maioria dos pacientes do sexo masculino, predomínio da raça branca, idade superior a 50 anos, uso freqüente de tabaco e álcool, predomínio do tipo histológico carcinoma espinocelular e estádios III e IV I $^{(4,6,7,14,15,29)}$.

No momento do diagnóstico a maioria dos pacientes relatou ingestão de pequenas quantidades de líquidos ou alimentos pastosos ou, então, não ingeriam nenhum tipo de alimentos, devido à disfagia rapidamente progressiva. Esse fator pode ocasionar deficiência de folato e elevação de homocisteína $^{(32)}$.

O período de sobrevivência dos pacientes foi semelhante ao observado na literatura, em que 56,2\% sobreviveram acima de 6 meses $^{(17)}$. A literatura sugere que a possível explicação para o baixo índice de sobrevivência pode ser o estado avançado da doença, bem como a debilidade orgânica desses pacientes, no momento do diagnóstico. $\mathrm{O}$ baixo nível sociocultural da população analisada contribui, de maneira importante, para o diagnóstico tardio e mau prognóstico dessa doença ${ }^{(9)}$.

No presente estudo, a anamnese nutricional mostrou que esses pacientes apresentavam comprometidos, embora o IMC tenha sugerido eutrofia ou sobrepeso na maioria dos indivíduos.

Por outro lado, o valor médio reduzido e a prevalência de hipoalbuminemia observada nesses pacientes podem indicar

TABELA 2. Comparação entre as médias e desvios-padrão e seus respectivos valores de $P$ das dosagens hematimétricas, bioquímicas e dos aminoácidos sulfurados entre os grupos de estudo e controle

\begin{tabular}{|c|c|c|c|c|c|c|c|c|c|c|c|}
\hline Variáveis & Ctl & $\mathrm{Hb}$ & $\mathrm{Ht}$ & Albumina & $\mathrm{Col} / \mathrm{Tot}$ & HDL & TGP & TGO & Tau & Cys & Hcy \\
\hline & $\mathrm{Cel} / \mathrm{mm}^{3}$ & $\mathrm{mg} \%$ & $\%$ & $\mathrm{~g} / \mathrm{dL}$ & $\mathrm{mg} / \mathrm{dL}$ & $\mathrm{mg} / \mathrm{dL}$ & $\mathrm{U} / \mathrm{L}$ & $\mathrm{U} / \mathrm{L}$ & $\mu \mathrm{mol} / \mathrm{L}$ & $\mu \mathrm{mol} / \mathrm{L}$ & $\mu \mathrm{mol} / \mathrm{L}$ \\
\hline G1 & $1592,2 \pm 548,8$ & $12,5 \pm 1,8$ & $39,2 \pm 5,6$ & $3,4 \pm 0,7$ & $157,1 \pm 28,1$ & $34,5 \pm 12,5$ & $30,0 \pm 13,6$ & $29,7 \pm 33,0$ & $123,6 \pm 77,5$ & $210,5 \pm 57,7$ & $18,7 \pm 7,3$ \\
\hline G2 & $1995 \pm 520,6$ & $45,8 \pm 15,2$ & $43,5 \pm 3,2$ & $4,2 \pm 0,4$ & $184,6 \pm 39,8$ & $45,8 \pm 15,2$ & $17,2 \pm 12,1$ & $10,9 \pm 7,2$ & $51,1 \pm 10,1$ & $278,2 \pm 35,1$ & $13,1 \pm 3,0$ \\
\hline$P$ & 0,046 & $<0,001$ & 0,006 & $<0,001$ & 0,005 & 0,022 & 0,005 & 0,018 & $<0,001$ & $<0,001$ & 0,004 \\
\hline
\end{tabular}

Teste $t$ de Student

$\mathrm{Gl}=$ grupo de estudo; $\mathrm{G} 2$ = grupo controle; $\mathrm{CTL}=$ contagem total de linfócitos; $\mathrm{Hb}=$ hemoglobina; $\mathrm{Ht}=$ hematócritto; $\mathrm{Col} / \mathrm{Tot}=$ colesterol total; $\mathrm{HDL}=$ colesterol; $\mathrm{TGP}=$ transaminase (alanina aminotransferase); $\mathrm{TGO}=$ transaminase (aspartato aminotransferase); Tau = taurina; Cys = cisteína; Hcy = homocisteína

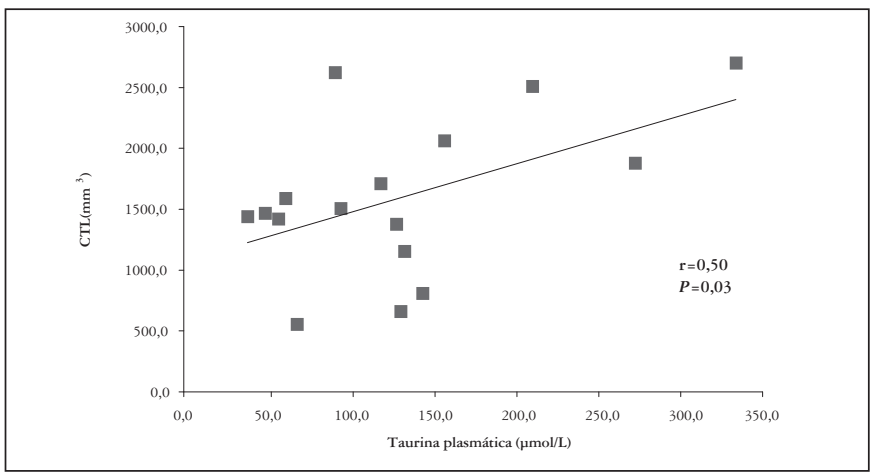

FIGURA 1. Correlação entre a taurina plasmática com a contagem total de linfócitos (CTL) dos pacientes

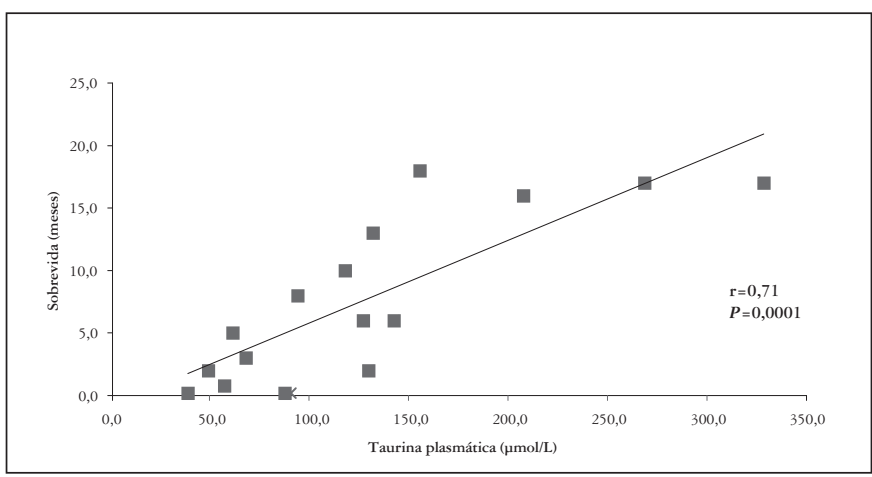

FIGURA 2. Correlação entre as concentrações de taurina plasmática com a sobrevida dos pacientes 
mau prognóstico para os mesmos. No entanto, FONSECA et al. ${ }^{(7)}$, analisando o resultado da derivação esôfago-gástrica em 24 pacientes com câncer avançado de esôfago, observaram que os valores da albumina plasmática não foram significantemente diferentes entre os pacientes com ou sem complicações póscirúrgicas.

A literatura sugere existir relação entre a alteração linfocitométrica com o aumento da mortalidade de pacientes $\operatorname{cirúrgicos}^{(2,9)}$. No presente estudo, não houve correlação estatística da sobrevivência pós-operatória com os níveis de linfócitos totais. Adicionalmente, a maioria dos pacientes não apresentou alterações significativas da CTL, em relação ao grupo controle. Resultado semelhante foi relatado por BELGHITTI et al. ${ }^{(2)}$, que estudaram 75 pacientes com câncer de esôfago. Esses autores observaram que a metade de seus pacientes não apresentou evidências de imunodeficiência mensurada por meio da contagem total de linfócitos.

Neste trabalho, o número de células do sistema imune (leucócitos, neutrófilos e linfócitos) mostrou-se dentro dos padrões de referência para pessoas normais mas, nos pacientes a CTL correlacionou-se positivamente com a taurina, sugerindo maior participação do sistema imune. Isso permite especular que a maior concentração de taurina no plasma desses pacientes poderia ser devido a maior necessidade orgânica para manutenção do sistema de defesa.

O grupo G2 apresentou concentrações plasmáticas dos aminoácidos sulfurados (taurina, cisteína e homocisteína), equivalentes às encontradas na literatura com referência para pessoas sadias ${ }^{(8,13,30)}$. A homocisteína foi elevada no grupo G1 e significativamente diferente do grupo G2. A hiperhomocisteinemia pode estar refletindo possível deficiência vitamínica, visto que esses pacientes no momento do diagnóstico, relatavam privação alimentar devido à disfagia, além de ser comum a incidência de hipovitaminose $\left(\mathrm{B}_{12}\right.$, folato e $\left.\mathrm{B}_{6}\right)$ em consumidores de álcool ${ }^{(3,20,30)}$. Embora haja evidências na literatura de que o aumento progressivo de homocisteína no plasma ocorra com a idade, principalmente em homens, essa alteração poderia ser devido ao declínio fisiológico na função renal e à diminuição da disponibilidade de vitaminas hidrossolúveis como folato, $\mathrm{B}_{6}$ e $\mathrm{B}_{12}{ }^{(32)}$.

Os níveis de taurina no grupo G1 mostraram-se elevados em relação ao grupo G2 $(P<0,05)$. Esse resultado poderia ser atribuído à mobilização desse aminoácido dos tecidos de maior capacidade de síntese e estoque, como fígado e músculo, para outros compartimentos como sistema nervoso central ${ }^{(8)}$. A elevação de taurina no plasma também pode ser decorrente da insuficiência renal, visto que os rins são os órgãos responsáveis pelo equilíbrio sulfurado no homem ${ }^{(26)}$; todavia, na presente casuística, a avaliação da função renal foi considerada satisfatória em todos os pacientes.

Outra possibilidade para o acúmulo plasmático de taurina seria sua menor excreção como taurocolato, que não foi avaliado neste estudo. Esse mecanismo justificaria o aumento plasmático desse aminoácido pela sua menor conjugação com ácidos biliares ${ }^{(12)}$.

Nesta série houve elevação dos níveis plasmáticos de taurina e correlação positiva da taurina com a sobrevida dos pacientes. Conforme o mencionado, a taurina participa efetivamente na preservação do fagócito e, portanto, na defesa do hospedeiro. Adicionalmente, correlacionou-se positivamente com linfócitos, também associados à defesa celular do hospedeiro. É sabida a importância da imunocompetência no prognóstico pósoperatório desses pacientes. Assim, de alguma forma ainda não esclarecida, a taurina elevada estaria associada a mecanismos de preservação e ao fortalecimento da imunocompetência de pacientes com esse tipo de câncer.

\section{CONCLUSÕES}

Assim, pacientes com CE em estágio avançado, mostraram elevações dos níveis plasmáticos de taurina e homocisteína e redução nos de cisteína e albumina. Os dois últimos são dependentes da ingestão protéica (total e de metionina) e estado pró-inflamatório e pró-oxidante.

Mesmo com a redução do precursor (cisteína) a taurinemia esteve elevada e associou-se positivamente com a contagem total de linfócitos e a sobrevida pós-operatória dos pacientes.

\section{Desdobramentos futuros}

Especula-se a importância do papel imunoprotetor da taurina na preservação da integridade dos fagócitos e na imunocompetência do hospedeiro contra o tumor.

Acredita-se que as alterações observadas no padrão de aminoácidos sulfurados desses pacientes possam ser sanadas com adequação protéico-sulfurado de metionina ou suplementos dietéticos de $\mathrm{N}$-acetilcisteína e, principalmente, com vitaminas hidrossolúveis do complexo $\mathrm{B}\left(\mathrm{B}_{2}, \mathrm{~B}_{6}, \mathrm{~B}_{12} \mathrm{e}\right.$ ácido fólico). 
Lamônica-Garcia VC, Marin FA, Lerco MM, Moreto F, Henry MACA, Burini RC. Plasma taurine levels in patients with esophagus cancer. Arq Gastroenterol. 2008;45(3):199-203.

ABSTRACT - Background - The esophagus cancer-host has a two way close relationship as seen in its sulphur-amino acid metabolism. Taurine one of these compounds has ubiquous role in host defense and other physiological mechanisms related to survival. Aim - To study the plasma levels of taurine and its precursors in patients with esophagus cancer. Methods - In a sectional design both groups, patients $(\mathrm{n}=16,43-73$ yrs old) and healthy controls ( $\mathrm{n}=20,27-65$ yrs old) were assessed for anthropometry, body-weight lost, hematology ( $\mathrm{Hb}, \mathrm{Ht}$, total leukocytes and lymphocyte counts), general biochemistry (albumin, glucose, lipids and aminotransferases) and chromatographic analysis for taurine, cysteine, and homocysteine. The survival time was registered there since from the clinical-histopathological diagnosis. All participants had a written ethical consent for the research. Results-The cancer patients were predominantly, white males of low social economic class, with spinocellular carcinoma stage IV located at upper 3 rd half of them presented hypoalbuminemia and $16 \%$ referred significant body-weight loss. The patients showed statistically lower values of Hb, Ht, total and HDL cholesterol and cysteine and significantly higher values of taurine, homocysteine and aminotransferases than healthy controls. A positive relationship was found between taurine and either TLC ( $\mathrm{r}=0.50)$ and survival $(\mathrm{r}=0.81)$. Conclusions - Lower plasma cysteine along with higher levels of taurine and homocysteine and the positive direct association of taurine with indications of survival suggest an effective role of this compound and therefore a prospective special nutritional care in its precursors (cysteine, methionine and B vitamins) of these patients.

HEADINGS - Esophageal neoplasms. Taurine

\section{REFERÊNCIAS}

1. Abe M, Takahashi M, Takeuchi K, Fukuda M. Studies on the significance of taurine in radiation injury. Radiat Res. 1968;33:563-73.

2. Belghiti J, Langonnet F, Bourstyn E, Fekete F. Surgical implications of malnutrition and immunodeficiency in patients with carcinoma of the oesophagus. Br J Surg. 1983;70:339-41

3. Borges-Santos MD, Burini RC. Bases metabólicas da suplementação de cisteína. Rev Bras Nutr Clin. 2005;20:259-64.

4. Cariati A, Casano A, Campagna A, Cariati E, Pescio G. Prognostic factors influencing morbidity and mortality in esophageal carcinoma. Rev Hosp Clin Fac Med São Paulo. 2002;57:201-4.

5. Condron C, Neary P, Toomey D, Redmond HP, Bouchier-Hayes D. Taurine attenuates calcium-dependent, fas-mediated neutrophil apoptosis. Shock. 2003;19:564-9.

6. Dietz J, Pardo SH, Furtado CD, Harzheim E, Furtado AD. Fatores de risco relacionados ao câncer de esôfago no Rio Grande do Sul. Rev Assoc Med Bras. 1998;44:269-72.

7. Fonseca CAM, Andrade-Sobrinho J, Pesciotto A, Rapport A. O tubo gástrico isoperistáltico no tratamento paliativo do câncer de esôfago. Rev Col Bras Cir. 2002;29:202-8.

8. Gray GE, Landel AM, Meguid MM. Taurine-supplemented total parenteral nutrition and taurine status of malnourished cancer patients. Nutrition. 1994;10:11-5.

9. Gurski RR, Schirmer CC, Rosa ARP, Santos WG, KrueL CD, Brentano L. Avaliação pré-operatória dos testes de hipersensibilidade retardada e da linfocitometria em pacientes com carcinoma epidermóide de esôfago. Rev HCPA Fac Med Univ Fed Rio G Sul. 1998;18:181-7.

10. Halawa I, Baig S, Qureshi GA. Use of high performance liquid chromatography in defining the abnormalities in the free amino acid patterns in the cerebrospinal fluid of patients with aseptic meningitis. Biomed Chromatogr. 1991;5:216-20.

11. Hansen SH. The role of taurine in diabetes and the development of diabetic complications. Diabetes Metab Res Rev. 2001;17:330-46.

12. Hayes KC, Sturman JA. Taurine in metabolism. Annu Rev Nutr. 1981;:401-25.

13. Jeevanandam M, Young DH, Ramias L, Schiller WR. Effect of major trauma on plasma free amino acid concentrations in geriatric patients. Am J Clin Nutr. 1990;51:1040-5.

14. Lopes MEA, Corona BEP, Cortés HE, Lizárraga LR. Cáncer del esófago: análisis de 167 casos del hospital general de México. Rev Méd Hosp Gen Mex. 1999;62:11-5.

15. Machado-Filho AC, Fedrizzi ER, Silva CAJ. Câncer de esôfago: análise de 52 casos. Arq Catarinenses Med. 1990;19:103-11.

16. Mahan LK, Arlin MT. Krause: alimentos, nutrição e dietoterapia. 8. ed. São Paulo: Roca; 1995.

17. Morais M, Paulo GA, Falcão J, Carvalho L, Manoukian NF, Rohr MRS. Terapêutica endoscópica paliativa de baixo custo no tumor avançado de esôfago: injeção de álcool, colocação de prótese plástica ou gastrostomia percutânea. GED Gastroenterol Endosc Dig. 2002;21:159-66.
18. Nonaka H, Tsujino T, Watari Y, Emoto N, Yokoyama M. Taurine prevents the decrease in expression and secretion of extracellular superoxide dismutase induced by homocysteine: amelioration of homocysteine-induced endoplasmic reticulum stress by taurine. Circulation. 2001;104:1165-70.

19. O'Flaherty L, Bouchier-Hayes DJ. Immunonutrition and surgical practice. Proc Nutr Soc. 1999;58:831-7.

20. Pelucchi C, Talamini R, Negri E, Levi F, Conti E, Franceschi S, La Vecchia C. Folate intake and risk of oral and pharyngeal cancer. Ann Oncol. 2003;14:1677-81.

21. Peskin AV, Winterbourn CC. Kinetics of the reactions of hypochlorous acid and amino acid chloramines with thiols, methionine and ascorbate. Free Radic Biol Med. 2001;30:572-9.

22. Redmond HP, Stapleton PP, Neary P, Bouchier-Hayes D. Immunonutrition: the role of taurine. Nutrition. 1998;14:599-604.

23. Schaffer SW, Takahashi K, Azuma J. Role of osmoregulation in the actions of taurine. Amino Acids. 2000;19:527-46.

24. Schuller-Levis GB, Park E. Taurine: new implications for an old amino acid. FEMS Microbiol Lett. 2003;226:195-202.

25. Stapleton PP, O'Flaherty L, Redmond HP, Bouchier-Hayes DJ. Host defense - a role for the amino acid taurine? JPEN J Parenter Enteral Nutr. 1998;22:42-8.

26. Suliman ME, Bárány P, Filho JC, Lindholm B, Bergström J. Accumulation of taurine in patients with renal failure [letter]. Nephrol Dial Transplant. 2002;17: 528-9.

27. Ubbink JB, Hayward Vermaak WJ, Bissbort S. Rapid high-performance liquid chromatographic assay for total homocysteine levels in human serum. J Chromatogr. 1991;565:441-6.

28. Ueland PM, Refsum H, Stabler SP, Malinow MR, Andersson A, Allen RH. Total homocysteine in plasma or serum: methods and clinical applications. Clin Chem 1993:30:1764-79.

29. Valladares GG, Tomasich FDS, Targa GZ, Gavarrete DD, Dias LAN. Câncer de esôfago. Estudo de 1292 casos tratados no período de 1990 a 2003. Rev Col Bras Cir. 2005;32:139.

30. Venâncio LS, Burini RC, Yoshida WB. Hiper-homocisteinemia na doença arterial periférica. J Vasc Bras. 2004;3:21-7.

31. Vinton NE, Laidlaw SA, Ament ME, Kopple JD. Taurine concentrations in plasma and blood cells of patients undergoing long-term parenteral nutrition. Am J Clin Nutr. 1986;44:398-404.

32. Zacarias-Castillo RR, Hernández-Rebollar AR, Zajarias-Rabchinskey A, GonzálezBárcenad D. Hiperhomocisteinemia. Um nuevo factor de riesgo coronariano. Gac Med Mex. 2001;137:335-45. 